\title{
Stephen Gill
}

\section{Das globale Panopticon Finanzwesen und Überwachung nach dem Kalten Krieg}

\section{Einleitung}

Am Beginn des 21. Jahrhunderts sind wir Zeuge eines Prozesses, in dem sich die neuen analytischen und informationsverarbeitenden Kapazitäten der Nachrichtendienste mit den neuen Ideologien nationaler Wettbewerbsfähigkeit verbinden. Darin drückt sich nicht nur eine partielle Abkehr vom Dogma des Kalten Krieges aus, sondern vor allem der Übergang in eine Periode zunehmender Globalisierung und eines disziplinierenden Neoliberalismus. Diese Entwicklung wurde 1991 von Admiral Stansfield Turner, dem CIADirektor der Carter-Administration, schon skizziert. Turners Bemerkungen nahmen bereits vorweg, welchen Nachdruck die bald folgende ClintonAdministration auf „ökonomische Sicherheit“ als ihre oberste politische Priorität legte. Clinton dehnte die nachrichtendienstliche Unterstützung der Vereinigten Staaten auf kommerzielle Organisationen aus, indem er neben dem Nationalen Sicherheitsrat einen neuen Nationalen Wirtschaftsrat schuf. Wie schon Turner formulierte:

... Wir leben in einem Informationszeitalter (...). Information war immer Macht, aber heute gibt es mehr Gelegenheiten, gute Informationen zu erhalten, und die Vereinigten Staaten haben bessere Möglichkeiten, das zu tun, als jede andere Nation (...). Die Sicherheit der USA wird jetzt vor allem in der ökonomischen Sphäre bedroht. Die Vereinigten Staaten haben sich von einer Hauptgläubigernation in den weltgrößten Schuldner verwandelt, und es gibt zahllose Industrien, in denen US-Firmen nicht mehr wettbewerbsfähig sind. Das bedeutet, dass wir im wirtschaftlichen Bereich ein besseres Nachrichtenwesen brauchen. Die Vereinigten Staaten wollen nicht von weltweiten Entwicklungen, neuen handelspolitischen Strategien, plötzlichen Rohstoffverknappungen oder unfairen und illegalen wirtschaftlichen Praktiken überrascht werden, die dem Land schaden. Es muß ein symbiotischeres Verhältnis zwischen der Welt der Nachrichtendienste und der des Business geben“ (zit. in Whitaker 1992: 126).

Der Zusammenhang, in dem diese Bemerkungen stehen, verweist auf die Verbindungen zwischen dem globalen Finanzsystem und politischer Macht im Kontext des „Informationskapitalismus“ und des wachsendem Tempo der Liberalisierung der globalen politischen Ökonomie. Nicht zuletzt, weil die zunehmende internationale Mobilität des Kapitals zu einer Krise der staatlichen Finanzsysteme führte, waren die Regierungen seit den 70er Jahren ge- 
zwungen, ein Investitionsklima zu schaffen, das nach globalen Standards als attraktiv beurteilt wurde (und oft entschieden die privaten Kreditprüfungsagenturen über die Kreditwürdigkeit der Regierungen), während sich zur selben Zeit Tendenzen zur Ausdehnung eines „transparenten Kapitalismus“ zeigten. Dies macht verständlich, warum viele Regierungen massiv in neue Technologien investiert haben, die den Aufbau und die Bearbeitung von Datenbanken ermöglichen, die zur Steuererhebung und im weiteren Sinn für Zwecke der sozialen Kontrolle und der Strafverfolgung eingesetzt werden können; aber niemand hat dermaßen viel investiert wie die USA. Vor diesem Hintergrund sollen hier neue Mechanismen öffentlicher und privater Überwachung oder - in der von Jeremy Bentham geprägten Terminologie - des „Panoptizismus“ (Bentham 1995) ${ }^{1}$ skizziert werden. Die Daten, die in diesem Prozeß gesammelt werden, werden instrumentalisiert: nicht nur für Zwecke der sozialen Kontrolle (also zur Stärkung der Macht des Staates), sondern auch zur Gewinnerzielung (und damit zur Stärkung der Macht des Kapitals). Dabei ist es natürlich für viele politische Führer und Institutionen eine Maxime der Klugheit, dass sie ihre eigenen Aktivitäten im Dunkeln halten, während andere einer intensiven Überwachung zugänglich sein sollen. Ein gutes Beispiel dafür ist der Internationale Währungsfonds.

In den neunziger Jahren verbanden sich die technischen Möglichkeiten zur Informationsgewinnung und -aufbereitung mit Konzeptionen der Wettbewerbsfähigkeit und eines disziplinierenden Neoliberalismus. Tatsächlich wurde die damalige Innovation der Überwachungspraxis und -technologie sowohl von staatlichen Apparaten - wie dem der USA - vorangetrieben, indem zu Zwecken der Planung, Steuererhebung und sozialen Kontrolle Informationen über die Bevölkerung und über legale wie über illegale Aktivitäten gesammelt wurden, als auch von kapitalistischen Unternehmen, die danach strebten, ihren Profit zu maximieren und/oder Risiken zu vermeiden oder zu begrenzen. Die verbesserten Möglichkeiten der Telekommunikations- und Softwaresysteme zur Sammlung, Zentralisierung und Analyse von Daten, die vielfach schwer zu bearbeiten waren oder früher geographisch oder funktional separat gespeichert wurden, gehören ebenfalls in diesen Kontext.

Das zentrale Argument dieses Essays ist, dass wir, wenn wir die Zunahme der „panoptischen“ Praktiken erklären wollen, diese in Beziehung zu den strukturellen Transformationen der globalen politischen Ökonomie am Anfang des 21. Jahrhunderts setzen müssen. Um dieses Argument genauer zu entfalten, werden im folgenden drei Hauptaspekte der panoptischen Praktiken und untersucht:

- öffentliche und private Überwachung im Informationskapitalismus: Verlustvermeidung und Profitmaximierung,

1 Für weitere Details siehe auch Gill (1995), Bauman (1998), Foucault (1993). 
- die kluge Führung der Marktakteure zur Minimierung von Systemrisiken in den Finanzmärkten,

- neue Architekturen der staatlichen Sicherheit: überwachender und disziplinierender Neoliberalismus.

Schließlich folgt eine Fallstudie über Finanzbetrug, Geldwäsche und politische Korruption.

Die Regime der nationalen und internationalen Regulierung der Ökonomie, werden nicht nur durch beschleunigte Kapitalbewegungen, sondern auch durch Handels- und Investitionsabkommen liberalisiert. Damit wird die „formelle“, d.h. die legale, steuerlich erfaßte ökonomische Welt zunehmend in legal-verbindliche Systeme einer globalen Regulierung und panoptischen Praxis eingeschlossen. Diese wiederum sind mit Bemühungen verbunden, den im System enthaltenen finanziellen Risiken zu begegnen und Verluste zu vermeiden, die durch Finanzinnovationen, größere Kapitalmobilität und Betrug verursacht werden. So sahen die neunziger Jahre das massive Wachstum der „informellen“, geheimen, oft illegalen „Offshore“-Ökonomie (vor allem den staatlicherseits kaum kontrollierten Banken und Fonds die in Freien Bankzonen angesiedelt sind, einschließlich der kaum regulierten kommerziellen Nutzung des Internets), die sich den der Regulierung und Besteuerung entzieht. Diese Offshore-Ökonomie umfaßt jedoch einen Komplex von ökonomischen Einheiten und Praktiken, der sowohl innerhalb der Jurisdiktion des staatlichen Systems operiert („Offshore“-Arrangements wie etwa freie Bankzonen beruhen auf der formalen juristischen Souveränität von Nationalstaaten) wie auch außerhalb der Reichweite seines intern geltenden Regelwerks.

Wenn diese Strukturen im globalen Finanzsystem kombiniert werden, begünstigen sie sowohl den legalen als auch den illegalen, den formellen wie den informellen Transfer von Ressourcen aus der Dritten Welt in die reichen Länder des Westens ebenso wie Geldwäsche, Finanzbetrug, Steuerflucht und andere Methoden, die dazu dienen, Wohlstand von den Armen zu den Reichen umzuverteilen. Dies zeigt unsere Fallstudie zu der in den frühen neunziger Jahren zusammengebrochenen „Bank of Credit and Commerce International“ (BCCI), die manchmal auch als „Bank of Crooks and Cocaine International“ (Internationale Gauner- und Kokainbank) bezeichnet wurde. Ein Bericht des US-Kongresses wies nach, wie die amerikanische Regierung und Sicherheitsbeamte internationale Finanzstrukturen ausplündern konnten, um Außenpolitik und (geheime) Kriegführung zu privatisieren. ${ }^{2}$

2 Über die dunklen Beziehungen zwischen der BCCI und der CIA scheinen nicht nur bewaffnete Konflikte, sondern auch die Teile der Außenpolitik der Reagan Administration finanziert worden zu sein, die vom Kongress und von Amerikas Verbündeten abgelehnt worden waren. 
Die BCCI war in den größten Bankbetrug und -zusammenbruch der Geschichte involviert. Vielfältige illegale Aktivitäten sowie die Manipulation von Finanzmärkten konnten ihr nachgewiesen werden: u.a. Fälle von Korruption und Bestechung, Drogengeschäfte, Geldwäsche, Waffenhandel ebenso wie der Einsatz von Prostituierten, um Freunde zu gewinnen und Menschen zu beeinflussen. ${ }^{3}$ Diese Bank hatte mit dem zu tun, was man als „diskretes“ oder auch korruptes Verhalten vieler politischer Führungspersonen bezeichnen kann. Dieser Umstand und die Rolle der CIA sind vielleicht zwei der Gründe, warum der BCCI-Skandal nie vollständig aufgeklärt wurde und wahrscheinlich auch nie aufgeklärt werden wird, waren doch so viele Personen in hohen Positionen beteiligt. In dieser Geschichte wurden die zentralen Regeln der Kapitalakkumulation zusammen mit den Grundsätzen des disziplinarischen Neoliberalismus unterminiert: die Glaubwürdigkeit der Regierungen, die Konsistenz der wirtschaftspolitischen Strategien und nicht zuletzt das Vertrauen der Marktteilnehmer und Investoren in die Finanzordnung. Daher könnte der Fall, den wir untersuchen - die BCCI - in einer Hinsicht recht verwirrend erscheinen, weil er einigen allgemeinen Tendenzen der achtziger und neunziger Jahre zu widersprechen scheint, die in diesem Essay herausgearbeitet werden: zum Beispiel, derjenigen, dass die nationalen Regierungs- und Sicherheitsapparate (z.B. der G7-Staaten) und die internationalen Finanzinstitutionen ein sehr hohes Maß an Überwachungskapazitäten erlangt haben. Der größte Bankenzusammenbruch der Geschichte enthüllte jedoch einen Mangel an effektiver Kontrolle und Regulierung seitens der Kommandohöhen des Finanzsystems, insbesondere was die amerikanischen und britischen Kontrollinstanzen betrifft. Er zeigte auch eine politische Doppelmoral. Vielleicht sind Skandale wie der BCCI-Zusammenbruch auch kennzeichnend für den Kapitalismus nach dem Kalten Krieg, wo die Politiker immer größere Geldmengen für ihre Wahlkampagnen und sie im Tausch für eine „Zusammenarbeit“ in Steuer-, Handels-, Investitions- und Regulierungsangelegenheiten erhalten.

\section{2. Öffentliche und private Überwachung}

„Panopticon“ ist ein aus dem Griechischen stammender Begriff, der die Bedeutung hat: „etwas, das alles sieht“. Foucault hat als Prinzip des Panoptizismus eine Überwachung beschrieben, die sowohl global als auch individualisierend ist. Benthams Entwurf für ein Gefängnis als Panopticon ist gut bekannt, obwohl es nie gebaut wurde. Es sollte durch das alles sehende und dominierende Auge des Aufsehers auf einem zentralen Wachtturm eine Transparenz sicherstellen, die

3 Einer der Dunkelmänner, dessen kriminelle Aktivitäten von einem Ausschuss des US-Senates 1992 aufgedeckt wurden, war der international operierende Finanzier Marc Rich. Dennoch begnadigte Präsident Clinton am Tag vor seiner Amtsübergabe den in der Schweiz ansässigen Rich. 
die Gefängnisinsassen dazu veranlassen sollte, Selbstdisziplin zu üben, d.h., sich gewissermaßen wie stellvertretende Aufseher zu verhalten. Das Panopticon setzte nicht voraus, dass jederzeit überwacht wurde, aber dass Überwachung jederzeit möglich war - eine Methode, die die Technologie der Überwachung nicht nur wirtschaftlich sinnvoll, sondern sie auch anwendbar machte, wenn es um die Kontrolle einer großen Bevölkerung ging.

Wie nicht nur Benthams, sondern auch Karl Polanyis Werk nahelegt, können hochentwickelte Formen staatlicher Kontrolle über die Bevölkerung notwendig sein, um eine auf individuellen Besitz gegründete, liberale Gesellschaft mit einem hohen Grad an ökonomischer Freiheit aufrechtzuerhalten. Bentham war ein Pionier im Erfinden von Systemen der Datensammlung durch Volkszählungen, der Zusammenstellung von Angaben über den Gesundheitszustand und über Verbrechen sowie im Hinblick auf seinen Kampf für immer rationalere Methoden, um wirtschaftliche Aktivitäten zu überprüfen und zu messen. Benthams soziale Innovationen sollten nicht nur produktiv sein, indem sie besser angepasste Mitglieder der Bevölkerung hervorbrachten, sie sollten auch profitabel sein (die ursprüngliche Idee für das Panopticon beruhte auf einer Fabrik in Rußland, die sein kapitalistischer Bruder Samuel Bentham leitete). Auf diese Weise nimmt die Idee des Panopticons sowohl die Entwicklung der modernen bürokratischen Systeme als auch die Verwendung technischer Innovationen der individuellen und massenhaften Überwachung und Datensammlung vorweg. Tatsächlich können auch einige Formen der Überwachungstechnologie recht nützlich sein, z.B. bei der Verifizierung von Vereinbarungen zur Rüstungskontrolle oder bei der Beobachtung ökologischer Prozesse (Verschwinden von Regenwäldern, Klimaveränderungen usw.).

Natürlich gibt es in keiner Gesellschaft ein einziges alles sehendes Auge (und das kann es auch nicht geben), und in der Tat verlangt eine demokratische Gesellschaft grundsätzlich einen gewissen Grad an Transparenz. Um effizient als Regulierungs-, Planungs- und Umverteilungsagentur funktionieren zu können, benötigt die Regierung bestimmte personenbezogene Daten. Allerdings ist der Umgang mit diesen Daten in der Praxis nicht immer demokratischer, öffentlicher Kontrolle unterworfen und gesellschaftlich akzeptiert.

In der OECD haben die Regierungen dem Aufbau umfassender und vernetzter Datenbanken große Aufmerksamkeit gewidmet. So ist z.B. die Praxis des Daten- (bzw. Akten-) Vergleichs (z.B. Vergleich von Sozialversicherungsakten mit Steuerakten, um Betrugsfällen auf die Spur zu kommen) in den USA und Kanada sehr verbreitet, in Japan und Großbritannien relativ üblich. Ein Teil der Aufmerksamkeit gilt auch der Zulässigkeit der Datenspeicherung - in Kanada ist diese Diskussion verstummt, während sie in Deutschland (wo der Datenabgleich stärker kontrolliert wird) wegen des Erbes der NaziVergangenheit wichtige politische Debatten hervorruft. Anderswo variiert der Grad öffentlichen Interesses. Australien, wie Kanada, Großbritannien oder die 
USA eine typische liberal-demokratische Gesellschaft des Lockeschen Typs, illustriert den allgemeinen Trend. Nach einer Krise des Staatshaushalts und der Veränderung der politischen und ökonomischen Landschaft von der Sozialdemokratie hin zu Konservatismus und Neoliberalismus erlangten Strafverfolgungs-, Steuer- und Wohlfahrtsbehörden wachsende Bedeutung innerhalb des Staatsapparats. Das Sozialministerium ging zu einer rigoroseren Überprüfung seiner Klienten über, einer Methode, die mit den Reformen verwandt ist, die Bentham für die Armenpflege befürwortet hatte und die die „Kontrollierbarkeit" von Ansprüchen und Verpflichtungen voraussetzt. Australien nutzt den Aktenvergleich, der durch die EDV wesentlich erleichtert wird. Das australische System wurde in die Datenbanken der Steuerverwaltung integriert (Davies 1995: 10-11). Ähnliche Entwicklungen können in Großbritannien beobachtet werden, wo Polizei, Sicherheitsdienste, Steuerverwaltung sowie Gesundheits- und Wohlfahrtsbehörden angeblich miteinander vernetzte Datenbanksysteme unterhalten. Das gesellschaftliche Klima, das eine solche Überwachung begünstigt, reflektiert auch die stark zunehmende Kriminalität (in diesem Fall besonders Kleinkriminalität, Vandalismus und Verbrechen gegen Personen), die in den meisten OECD-Ländern in den vergangenen zwei Jahrzehnten festzustellen war.

Einerseits freuen sich Regierungsbehörden (insbesondere Nachrichtendienste) über das Wachstum des Internets, weil es die Sammlung und Auswertung potentiell riesiger Mengen von (sensiblen) Informationen erlaubt. Auf diese Weise versucht der öffentliche Sektor private Datenbanken in seine Informationsstrukturen $\mathrm{zu}$ integrieren, um z.B. das Finanzsystem zu überprüfen, Steuerforderungen zu verfolgen und in einigen Fällen Betrug, Geldwäsche und „Staatsfeinde“ aufzudecken. Andererseits bauen private Informationsgesellschaften große Dateien mit personenbezogenen Daten auf, von denen ihnen wiederum viele von Regierungen verkauft wurden. In diesem Zusammenhang muß darauf hingewiesen werden, dass es in der Bundesverfassung der USA - wo sich die größten und aggressivsten Datenfirmen befinden keine Regelungen zum Schutz der individuellen Privatsphäre gibt. Die Kompetenz hierzu liegt bei den Einzelstaaten, was signifikante Unterschiede zuläßt oder - wie ein Journalist formulierte - ein System, das ,an eine Decke mit vielen Löchern“" erinnert (O'Harrow 1998b).

Obwohl nur wenige Bundesstaaten - wie etwa Maryland - ihre amtlichen Dokumente aktiv an Privatfirmen verkaufen, hat ,jeder Staat (...) seine Aktenmagazine Informationshändlern geöffnet, die Millionen von Dokumenten kopiert und in den Computer eingegeben haben." Maryland z.B. hat Dokumente verkauft, die Fahrerlaubnisse, Kraftfahrzeug-Zulassungen, Eigentumsverhältnisse und „personenbezogene Gerichtsverfahren“ betreffen. Die Datenbanken privater Firmen enthalten oft nicht nur Angaben über Kredite und manchmal auch den Gesundheitszustand, sondern auch „geheime Tele- 
fonnummern, Sozialversicherungsnummern, körperliche Details wie Größe und Gewicht sowie die Beschreibung und den Wert des Hauses, das die Person bewohnt" (O'Harrow 1998b). Diese Entwicklung hat in den USA zu einer riesigen Zahl von Beschwerden und Klagen geführt.

Dieses Beispiel ist wichtig, weil amerikanische (ebenso wie andere) Datenfirmen infolge der neuen Phase der Handels- und Investitionsliberalisierung, wie sie im GATS Abkommen angestrebt wird (Sinclair 2000), eine größere internationale Reichweite erlangen werden. So könnten wir wegen neuer Niederlassungsrechte für die Unternehmen eine Globalisierung des amerikanischen Musters erleben - unter einem neuen Regime „ökonomischer Freiheit“, das durch die Welthandelsorganisation WTO verkörpert wird. Nach den Erfahrungen der neunziger Jahre können wir durchaus annehmen, dass das amerikanische Kapital sehr darauf drängen wird, Liberalisierungsmaßnahmen durchzusetzen, welche die Expansion seiner privaten Datenfirmen unterstützt. Daher ist die entscheidende Frage hier die nach den Verbindungen zwischen den Handels- und Investitionsliberalisierungen, die in internationalen Vereinbarungen niedergelegt sind, und ihrer Ausdehnung auf Finanz- und andere Dienstleistungen auf der einen Seite und der Entformalisierung der globalen politischen Ökonomie in Gestalt der „Offshore“-Welt (von der das Internet eine Schlüsselkomponente ist) auf der anderen Seite. Tatsächlich scheint es so, dass der Geist schon aus der Flasche entwichen ist:

„Selbst wenn Behörden Informationen in Online-Datenbanken löschen wollen, ist das nicht immer möglich. In Texas bezahlte letztes Jahr eine Firma namens Public-Data.com 1.600 Dollar, um eine Datenbank mit Angaben über rund 14 Millionen Autofahrer und drei Millionen andere Personen mit in diesem Staat ausgestellten Personalausweisen zu kaufen, die sie ins Netz stellte. Aber als das texanische Parlament ein Gesetz verabschiedete (...), das jedermann, der Informationen über Kraftfahrzeuge und ihre Besitzer erwarb, zum Versprechen verpflichtete, die Daten nicht ohne Zustimmung der Betroffenen ins Netz zu stellen, verlegte Public-Data ihren Firmensitz einfach ins Ausland - auf die britischen Westindischen Inseln - und ließ die Informationen im Netz" (O'Harrow 1998b).

Abgesehen davon, dass dieses Beispiel zeigt, wie ein Mangel an Koordination und/oder Konkurrenz zwischen den Rechtsprechungen es Informationsunternehmern erlaubt, bestimmte Regeln zu umgehen und von ihrer relativen Mobilität zu profitieren, unterstreicht es auch die Schwierigkeit, öffentliche und private Aspekte von Datensammlung, Verschlüsselung und Überwachung zu unterscheiden. Es geht hier um die breiteren operativen Strukturen des modernen Kapitalismus und die Verwandlung oft sehr sensibler amtlicher Informationen in Waren. In der Tat haben viele private Agenturen nun große zentralisierte Datenbanken oder vielmehr Datenkaufhäuser, die öffentliche und private Informationen enthalten, die durch enorm leistungsfähige und immer schneller arbeitende Computer erlangt werden. Im vergangenen Jahrzehnt ist die Zahl dieser Datenkaufhäuser in den USA von 100 auf über 1000 angestiegen, was auch die Verzehnfachung der Zahl jener Firmen bedeutet, die sich 
dem gewidmet haben, was die Washington Post eine „Welle aggressiver Datensammlung" in den Jahren 1993-1998 genannt hat (O'Harrow 1998a: A1, A18). Daten werden von allen Transaktionen gesammelt, die elektronische Spuren hinterlassen, einschließlich e-mails, „Surfen“ im Internet, Verwendung von Geld- oder Kreditkarten. Eine der größten Firmen ist Experian (früher TRW), eine in den USA beheimatete Kreditprüfungs- und Marketingorganisation, die sich 1994 rühmte, detaillierte ökonomische und soziale Daten über 170 Millionen amerikanische Staatsbürger zu besitzen (bei einer Gesamtbevölkerung von damals ca. 254 Millionen Menschen). Im März 1998 behaupteten Manager von Experian, dass eine Datenbankrecherche, die 1994 auf einem gigantischen Großrechner noch sechs Minuten benötigte, jetzt nur noch 19 Sekunden dauern würde. Experian wurde 1996 zunächst für $1 \mathrm{Mrd}$. USDollar verkauft, sieben Wochen später erzielte die Firma beim Wiederverkauf bereits 1,7 Mrd. Dollar. Ungefähr $10 \mathrm{Mrd}$. Dollar jährlich werden in den USA nun ausgegeben, um private Datenkaufhäuser aufzubauen und zu erhalten (O'Harrow 1998a: A1, A18). Viele dieser Daten werden benutzt, um Markt- und Konsumentenprofile zu erstellen, wobei die Menschen nach ihrer wirtschaftlichen Bedeutung und/oder dem Risiko, das sie für das Unternehmen darstellen, eingeordnet werden.

\section{Panoptizismus als Mythos von Überwachung und Kontrolle}

Während die Produktion - wie Samuel Benthams Fabrik - in erheblichem Maße eine gegebene territoriale Basis und Arbeitskraft erfordert, sind Finanz- und Kreditwesen potentiell ziemlich mobil. Insbesondere in einer liberalisierten Ordnung sind sie viel freier von politischen und juristischen (räumlichen) Zwängen. Das mobile Finanzkapital ist daher fähig, Regierungen, die keine Kontrolle über das Finanzwesen aufrechterhalten, schnell zu bestrafen, indem Kredite verweigert werden, was es teurer macht, zu Geld zu kommen. Kreditprüfungen werden nicht nur auf Individuen angewandt, sondern auch auf Firmen und Regierungen, und sie helfen dabei, die Erwartungen der großen institutionellen Anleger auf den Märkten zu gestalten und eine Struktur von Macht und Wissen zu formen. Was die Regierungspolitik betrifft, so wirkt diese Struktur von Macht und Wissen als eine materielle Kraft, um Abweichungen von der orthodoxen Politik, wie sie im sogenannten „Washington-Konsens“ definiert ist, zu disziplinieren. Die Versuche der Weltbank, „good governance“ und eine Reform der staatlicher Strukturen der Dritten Welt zu erzwingen, indem sie ihre Kreditvergabe daran knüpft, sind das Mittel, um den „eisernen Gesetze der Ökonomie" (so ein Vertreter des IWF, zit. in O'Harrow 1998a: A1, A18) zum Durchbruch zu verhelfen. Das heißt, es soll den Empfehlungen der monetaristischen Marktorthodoxie gefolgt werden: Schuldenabbau, Währungsabwertung, Preisfreigabe, Freihandel, Reduktion des öffentlichen Sektors. 
So wurden z.B. 1978 die Vertragsartikel des IWF geändert, um eine weitergehende Überwachung der nationalen Wirtschaftspolitiken anhand eines Verhaltenskodex zu erlauben. Als diese Überwachungskapazität mit den Bedingungen verbunden wurde, die der IWF an seine Kreditvergabe knüpfte, verließ der IWF sein traditionelles Aktionsfeld, die makroökonomische Stabilisierung, und begann damit, besonders den Ländern der Dritten Welt, institutionelle Veränderungen ebenso wie mikroökonomische Reformen vorzuschreiben. Der IWF drängte entschieden auf Veränderungen in der Steuerpolitik, wobei er höhere indirekte Steuern und niedrigere Einkommens- und Körperschaftssteuern befürwortete, ebenso wie Regelungen des Konkursrechts und neue gesetzliche Bestimmungen zum Schutz von Investoren vor Enteignung - alles Maßnahmen, die darauf abzielen, die strukturelle Macht des Kapitals unter einem disziplinarischen Neoliberalismus zu stärken. Der IWF nutzt jetzt die Konditionalität seiner Kredite, um Institutionen und Politikern Anweisungen zu erteilen, wie sie das Bank-, Vertrags- und Gesellschaftsrecht einzurichten haben und welche Rolle gerichtliche Prüfungsmechanismen spielen sollen.

Die fundamentalen politischen und ökonomischen Ziele der G7 und der internationalen Finanzinstitutionen wurden jüngst von Michel Camdessus, dem früheren Direktor des IWF, in einer Rede ausgesprochen, die er im März 1999 vor dem Institute of International Bankers in Washington hielt. Camdessus entwarf eine neoliberale Utopie für das 21. Jahrhundert: ein Globalisierungsprojekt, das „die universelle Förderung von freien Marktmechanismen, gestärkt durch eine Reihe von Standards und Prinzipien des richtigen Verhaltens“ beinhalten sollte. Insbesondere rief Camdessus nach „offenen und integrierten Kapitalmärkten, die durch einen allmählichen, durch gute makroökonomische Politik und gesunde Finanzinstitutionen unterstützten Liberalisierungsprozeß zu erreichen" seien. Des weiteren skizzierte Camdessus eine Welt, in der das Kapital („der private Sektor“) nach rationalistischen Prinzipien handeln würde, gestützt auf den vollständigen Zugang zu den relevanten öffentlichen und privaten Informationen, mit Datenbanken, die nach einheitlichen Standards des Finanz- und Rechnungswesens aufgebaut wären, so dass Risiken effektiver eingeschätzt werden könnten. Tatsächlich faßte Camdessus jenen Prozeß der Normierung und Überwachung ins Auge, den wir „Panoptizismus“ nennen:

„Eine Welt, in der Marktteilnehmer und Regierungen nach hohen Standards von Transparenz und Führung handeln werden (...) Eine Welt, die in erster Linie auf den privaten Sektor - sowohl den einheimischen als auch den ausländischen auf wahrhaft gleicher Grundlage - bauen wird, um Ressourcen für Investition und Wachstum zu mobilisieren. All das beinhaltet, dass es eine Welt sein wird, in der Märkte in der Lage sein müssen, effizient zu funktionieren, mit realistisch eingeschätzten Risiken und gerecht verteilten Belohnungen von Erfolgen und Kosten von Niederlagen." (Camdessus 1999) ${ }^{4}$

4 Camdessus bemerkte weiter, dass eine universelle Erweiterung der Märkte einer soliden Wirtschaftspolitik bedürfe und, dass auch ausgleichende Maßnahmen notwendig seien, um 
Es verdankt sich diesen Forderungen, dass der Diskurs des disziplinarischen Neoliberalismus zunehmend die staatliche Politik beeinflußt hat. In gewissem Umfang ist die staatliche Politik durch die „drei C“ neu definiert worden: Regierungen messen ihre Glaubwürdigkeit (credibility) und die Konsistenz (consistency) ihrer Politik daran, inwieweit sie das Vertrauen (confidence) der Investoren erhalten. Wenn Regierungen das Vertrauen der Investoren verlieren, flieht das Kapital in sichere Häfen. In einer Welt des disziplinarischen Neoliberalismus sind die „drei C“ die Maximen richtigen Verhaltens im Kontext der allmählichen Globalisierung der amerikanischen Auffassung eines „transparenten Kapitalismus“ (Braithwaite/Drahos 2000). So definiert z.B. die Weltbank Glaubwürdigkeit als die „Zuverlässigkeit des institutionellen Systems“ und „die Berechenbarkeit ihrer Regeln und Politiken sowie die Folgerichtigkeit, mit der sie angewandt werden", wobei es hauptsächlich um die Notwendigkeit geht, Unsicherheit in den Überlegungen von Investoren zu vermindern; andernfalls halten Investoren solche Staaten nicht für glaubwürdig (World Bank 1997: 4-5).

Auf diese Weise sind zumindest in der Theorie alle Politikfelder, die unternehmerische Risiken und Profitraten betreffen, einer konsistenten Überwachung („Transparenz“), Prüfung und Bewertung nicht nur durch Regierungsbehörden, IWF und Weltbank unterworfen, sondern auch durch private Stellen wie Firmen und Ratingagenturen wie etwa Moody's. Dies schafft eine Form von makro- und mikroökonomischer Disziplinierung von Regierungen und Wirtschaftsakteuren, die dazu tendiert, die Macht des mobilen Kapitals in Staat und Zivilgesellschaft noch stärker zu befestigen.

Um diesen Zustand zu ermöglichen, ist nicht nur eine begrenzte, verfassungsmäßige Regierungsform erforderlich (die den Staat einer Zivilgesellschaft unterordnet, die von den großen Eigentümern dominiert wird), sondern auch ein starker Staat, der über die Mittel verfügt, bestimmte Praktiken als legal zu fördern und andere als illegal zu verfolgen. Darüberhinaus muß dieser Staat über die Kapazitäten zur Überwachung und Repression verfügen, um soziale Unruhen und die verschiedenen Formen politischen Widerstands einzudämmen, die durch eine schnelle Liberalisierung unter den Bedingungen der sich intensivierenden Globalisierung hervorgerufen werden. Gleichzeitig werden Anstrengungen unternommen, die politische Opposition einzubinden und die Marktgesellschaft zu legitimieren. Die Entwicklung einer Konsumentenkultur, die sämtliche Bedürfnisse nur noch durch Waren befriedigt sowie die Herausbildung einer massenhaften Anlegerkultur, die an den Herzschlag der internationalen Aktien- und Geldmärkte gebunden ist, sind wesentliche Mit-

mit Marginalisierungen und sozialen Umbrüchen umgehen zu können. Somit ist die Utopie des IWF eine Welt, in der „kein Land ausgegrenzt wird ... mit einer Sozialpolitik, die gleichberechtigte Bildungsmöglichkeiten, Gesundheitsfürsorge und die Aussicht auf einen gesicherten Lebensunterhalt ermöglicht und die sozialen Schutz in Krisenzeiten gewährleistet.“ 
tel dafür. Diese Methoden wiederum sind mit staatlichen Politiken verknüpft, die dem utilitaristischen Prinzip des größten Glücks der größten Zahl folgen (auch wenn das eine sehr kleine Minderheit der Weltbevölkerung ist). Hierzu sind Systeme erforderlich, mit denen (etwa durch Volkszählungen) Bevölkerungen klassifiziert und organisiert werden können und ihre Aktivitäten gemessen werden können. Schon für Jeremy Bentham war der bevorzugte Ausdruck für öffentliche Verwaltung und Produktion „Kontrollierbarkeit“. In Benthams Verständnis sollte sich der Staat - wenn nicht in der Praxis, so zumindest der Theorie nach - in die Zivilgesellschaft einmischen, um dabei zu helfen, das „allgemeine“ Glücksziel der Utilitaristen zu fördern. Das implizierte die Notwendigkeit, die soziale Ordnung zu sichern und eine Struktur sozialer und politischer Überwachung zu schaffen, um das Verhalten zu beeinflussen und „gute Taten“ (Foucault 1993) zu unterstützen. Für Camdessus bedeutet dies, im Rahmen der „drei C“ des Kapitals zu handeln.

\section{G7-Nexus, liberalisiertes Finanzwesen und vorbeugende Überwachung}

In den globalen Machtkonstellationen stehen die Vereinigten Staaten im Zentrum der transnationalen Strukturen, die vom „G7-Nexus“ bestimmt werden, dessen Maximen guten Verhaltens auf die obenerwähnten „drei C“ gegründet sind. In diesen Nexus sind Finanzminister, Präsidenten von Zentralbanken und internationale Netzwerke von Wertpapierverwaltern ebenso eingebunden wie „private“ Foren der Meinungsbildung wie etwa das Weltwirtschaftsforum. Finanzielle Regulierung und Koordination beruhen auf der Praxis der „gegenseitigen Überwachung“, obwohl dominante Interessen - z.B. die der USA - offenkundig den Ausschlag geben. Schätzungen und Vorschläge der G7 werden vom IWF, der Berichte über die Weltwirtschaft verfaßt, geprüft und kritisiert. Diese Ergebnisse werden auch mit denen der OECD verglichen ebenso wie mit den Untersuchungen und Angaben privater Organisationen und Gremien wie der Gruppe der 30 (die sich mit Finanzinnovationen und Überwachung beschäftigen), von einzelnen Firmen wie Moody's, Standard \& Poor's und IBCA (deren Aufgabenfeld die Prüfung der Kreditwürdigkeit von Unternehmen und Regierungen ist) sowie großen Akteuren in den Währungsmärkten (hauptsächlich institutionelle Investoren - Fonds und Versicherungsgesellschaften - oder vermögende Spekulanten wie George Soros). In diesen $\mathrm{Zu}$ sammenhang gehört auch die Schaffung neuer internationaler Datenstandards und Buchführungsmethoden, juristischer und institutioneller Rahmenbedingungen. Im Ergebnis setzt die Effektivität und Präzision der Politik aktuelle und verwertbare Daten voraus, die nach einheitlichen Regeln erhoben werden.

Gleichzeitig hat die Bedeutung einer vorbeugenden Überwachung enorm zugenommen, da die Liberalisierung des Finanzsystems eine stärkere Spekulati- 
on begünstigt und mit den unsteten Schwankungen von Preisen, Wechselkursen und Zinsraten erhebliche Risiken verbunden sind. In mancher Hinsicht scheint das internationale Finanzsystem unterreguliert und von einem Kollaps bedroht zu sein, denn es ist nur so stark wie sein schwächstes Glied. Dies zeigte sich z.B. 1998, als das auf den Cayman Islands angesiedelte Long Term Capital Management (ein obskurer, US-Normen nicht unterworfener Fonds) fast die gesamte Wall Street ins Wanken gebracht hätte - trotz der Bemühungen der G 10, angesichts der Schuldenkrise der Dritten Welt und unvorsichtiger Kreditvergabe durch Privatbanken zu einer Harmonisierung der Standards angemessener Kapitaldeckung und anderen vorbeugenden Maßnahmen zu gelangen. Daher wird die Liberalisierung des Finanzwesens, die für die vergangenen zwei Jahrzehnte charakteristisch ist, in gewissem Umfang auch von einem Konsolidierungskurs begleitet, wenn etwa die Bank für Internationalen Zahlungsausgleich und die nationalen Zentralbanken versuchen, ihre Aufsichtsbefugnisse im Rahmen eines sich quasi selbst regulierenden Modells auszudehnen, in dem international tätige Geschäftsbanken selbst eine globale Risikoanalyse erstellen müssen, um Zahlungsausfälle und finanzielle Zusammenbrüche zu vermeiden, statt sich auf die Aufsicht des Gastlandes zu verlassen (Braithwaite/Drahos 2000). Zur selben Zeit und trotz dessen, was in der Asienkrise 1997 manifest wurde, kündigte der Internationale Währungsfonds Pläne zur Veränderung seiner Vertragsartikel an, um sich auf diese Weise aktiver für die Beseitigung von Restriktionen der Kapitalmobilität und andere Formen der Liberalisierung einsetzen zu können. ${ }^{5}$

Eine Erklärung für diese Tendenzen sind darin zu sehen, dass in einer Reihe von Staaten immer größer werdende Blocks von Rentiers in der Finanz- und Wirtschaftspolitik die Oberhand gewonnen haben (Gill 1998). Das ist in einem Meinungsklima geschehen, das zunehmend eine kurzfristige, ökologisch kurzsichtige „Schnell-reich-werden-Mentalität“ begünstigt hat. Eine der Folgen davon ist, dass Manager von Unternehmen wie auch von staatlichen Pensionsfonds zweifelhafte Risiken mit Produkten eingehen, die sie nicht verstehen. ${ }^{6}$ Zusammenfassend läßt sich sagen, dass die Regierungen mit der Zunahme der internationalen Mobilität des Kapitals gezwungen waren, den „transparenten Kapitalismus“ auszudehnen und in Datenbanken zur Steuererhebung,

5 In ähnlicher Weise suchen auch große Investoren, wie Pensionsfonds und Versicherungsgesellschaften, die in den vergangenen zwanzig Jahre ein enormes Wachstum verzeichneten, eine „best practice“ der Regulation und Veröffentlichungspflicht. Mit den vielen Lohnabhängigen, die später einmal Renten aus diesen Institutionen beziehen werden und daher an ihrem ökonomischen Erfolg interessiert sind, hat sich der Kreis der „Investoren“ politisch und wirtschaftlich erheblich erweitert.

6 Orange County in Kalifornien erlebte am 6. Dezember 1994 mit einem Verlust von 1,7 Mrd. Dollar den größten Bankrott in der Geschichte. Der Zusammenbruch scheint durch ungesicherte Spekulationen an Finanzmärkten des Finanzbeauftragten des Counties zustande gekommen sein. Für weitere Detail siehe die Business Week vom 13.3.1995. 
sozialen Kontrolle und Strafverfolgung zu investieren. Als die sogenannte ,informelle" Ökonomie expandierte, dehnten die Staaten ihrerseits die Überwachung - auch die der „Offshore-Ökonomie“ - aus. Auf der anderen Seite bedrohen die verstärkte Kapitalmobilität, Finanzinnovationen und Betrug die Integrität des globalen Finanzsystems und machen daher eine vorbeugende auch kriminalistische Überwachung notwendig, um Bankbetrug, Zahlungsausfälle und finanzielle Zusammenbrüche zu vermeiden. Darüberhinaus müssen Zentralbanken und internationale Finanzinstitutionen verstärkt als „lender of last resort" fungieren, um die Effekte der ökonomischen Krisen zu minimieren, die seit den siebziger Jahren häufiger geworden sind. Wie jedoch der BCCI-Fall zeigt, können vorbeugende Überwachung und Strafverfolgung unzureichend sein, um Geldwäsche und Finanzbetrug zu verhindern - denn diese Phänomene können integraler Bestandteil der politischen Existenzbedingungen der gegenwärtigen Finanzordnung sein.

\section{5. „Future Image Architecture": die Überwachung von Feinden und Freunden}

Zur Ära nach dem Kalten Krieg gehört die beschleunigte Entwicklung des militärischen und politischen Überwachungsapparats, der eine Welt kontrolliert, in der sich soziale Ungleichheit, politische Polarisierung und geschäftliche Rivalität intensivieren und sich die Ausbreitung von Waffen fortsetzt. Daher wäre dieser Essay unvollständig, würde er nicht einen Bereich erwähnen, der im konventionellen Verständnis Überwachung repräsentiert: die Welt der Spionage und der Geheimdienste, die John Le Carré so überzeugend porträtiert hat und die die systematische Sammlung von Informationen durch Staaten über andere Staaten und Bürger bezweckt. Das Ziel der Nachrichtenapparate deckt sich mit dem modernen Mythos, der uns vorher schon im Hinblick auf den IWF begegnet ist: sowohl Freunde als auch Feinde unter totaler Kontrolle zu halten.

Anläßlich der Berichterstattung über das in der Volksrepublik China im April 2001 notgelandete amerikanische Spionageflugzeug erzählte ein Veteran des US-Marinegeheimdienstes Journalisten, ein Offizier seiner Einheit habe Visitenkarten mit dem Aufdruck: „Auf Gott vertrauen wir. Alle anderen kontrollieren wir" (Drew 2001: A5). Das ist durchaus zutreffend, da es jetzt die Sowjetunion nicht mehr gibt, und weil die ökonomischen Herausforderungen für die US-Vorherrschaft schwerer einzuschätzen sind als es mit der sowjetischen Bedrohung der Fall war. Jetzt sind selbst „Freunde“ der USA ein möglicher Gegenstand der Überwachung, jedenfalls wenn man den USSicherheitsbehörden, Theoretikern des Nachrichtenwesens und Planungsdokumenten des Pentagon Glauben schenkt. Es ist kaum nötig zu sagen, dass das in der Europäischen Union und anderenorts Bedenken hervorgerufen hat (vgl. Wright 1998; Europa-Parlament 1999; Bronskill 2001). 
Während des letzten Jahrzehnts konnte der Militär- und Sicherheitsapparat der Vereinigten Staaten seine prominente Position und relative Autonomie innerhalb des Staates erhalten, indem er sich stärker auf das wirtschaftliche Nachrichtenwesen konzentrierte und diejenigen überprüft, maßregelt und sanktioniert, die Grundprinzipien des disziplinarischen Neoliberalismus in Frage stellen. Das ist bemerkenswert, weil der US-amerikanische Überwachungsapparat nicht immer auf eine Weise tätig gewesen ist, die mit dem neoliberalen Stil des freien Marktes vollkommen übereinstimmt. Vielmehr übt der Nachrichtenapparat nicht nur enorme politische Macht aus, sondern „zeigt auch die meisten der typischen Attribute großer bürokratischer Organisationen, einschließlich einer Tendenz zur Definition und Verfolgung bürokratischer politischer Ziele, die nicht unbedingt voll mit den nationalen Interessen der angelsächsischen Länder übereinstimmen“ (Jeffrey/Ball 1990: 301).

Die Infrastruktur des US-Nachrichtenapparats entstand während des Zweiten Weltkriegs unter der Leitung und Ausbildung durch den britischen MI6 in Gestalt des Office of Strategic Services (OSS). Sie wurde international durch die sogenannten „UKUSA agreements“ gestärkt, die die Geheimdienste der größten englischsprachigen Länder (und anderer, die später hinzukamen) an den der USA banden und ihm unterordneten, wobei die Verbindung zwischen dem Vereinigten Königreich (UK) und den Vereinigten Staaten (USA) das Herzstück bildete:

„Die UKUSA agreements von 1947 waren die Gründungsurkunde eines globalen Netzwerks ausgeklügelter elektronischer Lauschtechnologie, aber zwischen den Zeilen dieser Vereinbarungen stand die institutionalisierte Hegemonie des amerikanischen TECHINT (technologically obtained intelligence) mit seinem Nervenzentrum in Fort Meade, Maryland, dem Hauptquartier der NSA“" (Whitaker 1992: 119).

Eine der bedeutendsten Behörden und die einzige, die nicht der Aufsicht durch den Kongreß unterliegt, ist die National Security Agency (NSA) mit angeblich 21.000 Mitarbeitern, die in ihrem Hauptquartier in Fort Meade, Maryland, stationiert sind (Bronskill 2001). Sie ist „der größte und geheimste Nachrichtendienst“, „das Herz des Systems“, „der innere Zirkel der Geheimnisse“ (Woodward 1987: 22; 46-47). Die NSA konzentrierte sich ursprünglich auf militärisch relevante Informationen, sammelt mittlerweile aber Informationen aller Art (Richelson/Evans 2001). ${ }^{7}$ Die NSA wurde 1998 Amerikanern und anderen besser - wenn auch in etwas umstrittener Weise - bekannt, als sie in den Mittelpunkt des Hollywoodfilms Enemy of the State gestellt wurde, mit Will Smith und Gene Hackman in den Hauptrollen und unter der

7 Das freigegebene Statut der NSA zeigt auch, zu welchen neuen Organisationsstrukturen es nach Ende des Kalten Krieges gekommen war. Während des Kalten Krieges gab es drei regionale Schlüsselgruppen: A (der Sowjet-Block), B (das kommunistische Asien) und C (alle anderen). Nach dem Zusammenbruch der Sowjetunion wurden diese Gruppen auf zwei reduziert: eine für Europa, eine für alle restlichen Nationen. Die neue Struktur ist gleichfalls Ausdruck für die Zunahme transnationaler Aktivitäten. 
Regie von Tony Scott. Dieser Orwell'sche Film zeigte, wie die verschiedenen Überwachungsformen - die oben erwähnten Datenbanken der Regierungen und Konzerne plus Ausnutzung der Telekommunikationsmittel, Satellitenüberwachung und andere Formen der Ausspähung von Zielen - durch den Einsatz von Hochgeschwindigkeitscomputern integriert und ausgewertet werden. Die durch den Film ausgelöste Unruhe führte zu einem seltenen öffentlichen Auftritt des NSA-Direktors, Generalleutnant Michael Hayden, der sich bemühte, dem Kongreß zu versichern, dass geeignete Schutzmaßnahmen vorhanden seien, um das Ausspionieren von US-Bürgern zu verhindern. Er bestritt auch, dass die NSA nachrichtendienstlich erworbene Informationen an US-Firmen weitergebe, und bezeugte, dass die NSA kein anderes Land ersuchen darf, „illegal“ Informationen über US-Bürger für die NSA zu sammeln (Richelson/Evans 2001).

Vielleicht am eindrucksvollsten in Enemy of the State war, wie elektronische Datenbanken in Verbindung mit detaillierten, von Satelliten gesendeten Überwachungsbildern genutzt wurden, um Verdächtige in Echtzeit zu verfolgen. In der Tat wurde im April 2001 bekannt gegeben, dass das geheime National Reconaissance Office (Nationales Erkennungsbüro) der USA von der Clinton-Administration und dem Kongreß ermächtigt worden war, sein System von Spionagesatelliten massiv zu erweitern, z.T. weil andere Staaten wie Rußland, Frankreich, Indien und enge amerikanische Verbündete wie Israel und Kanada Satellitenüberwachungssysteme unterhalten. Die US-Initiative zieht das teuerste Vorhaben nach sich, das je von amerikanischen Nachrichtendiensten unternommen wurde, nämlich die „Future Image Architecture“ (FIA). Diese wird im Verlauf von 20 Jahren 25 Mrd. US-Dollar kosten; zum Vergleich: das Manhattan-Projekt zum Bau der Atombombe während des Zweiten Weltkriegs kostete (nach heutiger Kaufkraft) $20 \mathrm{Mrd}$. Dollar. Die neuen Systeme sollen andere Überwachungssysteme ergänzen, um den USA eine nur ihr zukommende Kapazität für bestimmte Arten von Militäraktionen zu verschaffen. Darüber hinaus hat es den Anschein, dass das neue System gut zur Entwicklung der militärischen Nutzung des Weltraums paßt, die für die Bush-Administration Priorität hat. Das neue Satellitensystem wird in $3.200 \mathrm{~km}$ Höhe stationiert werden, acht- bis zwanzigmal mehr Bildmaterial und Details sammeln als die gegenwärtigen Systeme und es wird schwieriger sein, ihm zu entgehen. Es wird fähig sein, jedes Ziel etwa 30 Minuten lang im Blickfeld der Kamera zu halten (gegenüber der jetzigen Begrenzung auf rund zehn Minuten), es wird genauer sein und „in der Lage, Objekte von der Größe eines Baseballs überall und zu jeder Zeit auf dem Planeten zu verfolgen“ (Fitchett 2001:1). Die New York Times stellte fest:

„Die wahrhaft revolutionäre Neuerung findet jedoch nicht am Himmel statt, sondern in den Computern der Bodenstationen, die das heruntergeladene elektronische Bildmaterial speichern, die Daten in nachrichtendienstlich brauchbare Informationen verarbeiten und die Bilder dann 
an eine wachsende Schar von US-Regierungsbehörden verteilen - die offiziellen Konsumenten des Materials (...). (Hierzu gehören auch) exakte Aufnahmen von Kriegsschauplätzen (...). Bislang dauerte es Tage, bis diese hoch aufgelösten Bilder, die auch bei Nacht oder durch Wolken aufgenommen werden können, bis hinunter zu den Offizieren gelangten“" (Fitchett 2001: 4).

Es ist sinnvoll, den Leser daran zu erinnern, dass im gegenwärtigen globalen Überwachungssystem, das von der NSA koordiniert wird, den USA alle „rohen" nachrichtendienstlichen Daten ihrer Juniorpartner zur Verfügung stehen, wofür sie im Tausch „bearbeitete“ Daten zurückgeben, was einige Vorteile für die Partner hat, insofern sie auf diese Weise ein fertiges Produkt erhalten. Zugleich gestattet es aber den US-Behörden, ihre Sicht der Welt und ihre Interpretation bestimmter Entwicklungen und Trends einfließen zu lassen (Whitaker 1992: 119).

Das Budget der NSA ist um ein Mehrfaches größer als das der CIA, obwohl ihre Gesamtleitung der Autorität des CIA-Direktors untersteht (Woodward 1987: 478). Wie erwähnt, ist die NSA auf elektronische Abhörmethoden spezialisiert, sowohl von großen Horchposten aus, die rund um die Erde verteilt sind, als auch in kleinen Teams, die in Botschaften stationiert sind, wobei Daten von Satelliten, Spionageflugzeugen und aus der Luftüberwachung genutzt werden wie auch eine Reihe exotischer technischer Erfindungen, um Zugang zu den Informationen und Gedanken anderer zu erhalten, seien sie nun „Freunde“ oder „Feinde“. So ist etwa die NSA-Bereichsstation F83 in Menwith Hill bei Harrogate (Yorkshire, England) die größte elektronische Spionagebasis in der Welt. Sie zapft unmittelbar das Mikrowellen-Netzwerk der British Telecom an (Bernal 2001). F83 hört die europäische und transatlantische Telekommunikation ab und arbeitet im Tandem mit der, streng geheimen Lausch- und Entschlüsselungseinrichtung der britischen Regierung, GCHQ in Cheltenham, zusammen. Eine dritte bedeutende Einrichtung dieser Art befindet sich in Pine Gap (Australien), andere sind in Seattle, Puerto Rico, Dänemark, West Virginia, Neuseeland, Zypern und Holland. Eine andere Bodenstation für das amerikanische SIGINT befindet sich in Bad Aibling in Deutschland.

Diese Stationen sind im sogenannten ECHELON-System zusammengefaßt, das die Daten in riesige Computer eingibt, die als Wörterbücher (Dictionaries) bezeichnet werden und schriftliche Mitteilungen automatisch auswerten, indem sie Listen von gesuchten Zahlen, Themen und Schlüsselwörtern verwenden. ${ }^{9}$ ECHELON ist vornehmlich für nichtmilitärische Ziele bestimmt:

8 Trotz vieler Unzulänglichkeiten (z.B. überschätzte der CIA in den siebziger und achtziger Jahren ständig die militärische Stärke der Sowjetunion; die schlechte Zusammenarbeit von CIA und NSA resultierte in einem mangelhaften Nachrichtensystem im Iran vor der Revolution von 1979) kontrollierte dieses Nachrichten-Netzwerk praktisch ganze Regierungen in der Dritten Welt (z.B. in den 80er Jahren Habré im Tschad, Zia in Pakistan, Doe in Liberia, Marcos auf den Philippinen, Nimeri im Sudan, Gemayel im Libanon, Duarte in El Salvador).

9 Der Spezialist des US Nachrichtendienstes Jeffrey Richelson bewies anhand von Akten der 
Regierungen, Unternehmen, andere Organisationen und Individuen. Da es in den meisten Staaten verboten ist, die eigenen Bürger auszuspionieren, erlauben die Vereinbarungen der UKUSA-Länder dies zu umgehen, indem z.B. „Briten Amerikaner und Amerikaner britische Staatsbürger ausspionieren und die beiden Gruppen die Daten austauschen“ (Bernal 2001).

ECHELON bringt die bisher getrennt arbeitenden Systeme zusammen und entwickelt sie weiter, um ein integriertes globales Netzwerk zu bilden, das dieselbe Ausrüstung und dieselben Methoden verwendet, um (oft auf unerlaubte Weise) Informationen zu gewinnen. Milliarden von Botschaften werden jeden Tag analysiert, z.B. mithilfe von Datenerhebungssoftware, die über Internetserver eingesetzt wird. ECHELON existiert schon seit den siebziger Jahren, wurde aber zwischen 1975 und 1995 erheblich erweitert. Vor ECHELON wußten die verschiedenen nationalen Sicherheitsbehörden und stationen, welche Mitteilungen abgehört wurden und an wen sie geschickt werden. Jetzt sind die Systeme viel stärker automatisiert und können eine viel größere Datenmenge bewältigen. Das bedeutet, dass potentiell alle Botschaften, die von „Wörterbuch“-Computern ausgewählt werden, automatisch an die NSA oder andere Nutzer weitergeschickt werden, ohne vorher vor Ort gelesen zu werden.

Trotz ihrer ausgedehnten Überwachungskapazitäten im Luft- und Weltraum wird die NSA wahrscheinlich Schwierigkeiten bekommen, ihren Zugriff auf die landgestützten Kommunikationsmittel zu behalten, weil es einen Wandel hin zu optischen Netzwerktechniken gibt; das Abfangen von Nachrichten ist dann nur mit einem physischen Zugang zu diesen Netzen möglich, und andere Staaten können ihre Mitwirkung verweigern. Darüber hinaus entwickeln nicht nur Regierungen, sondern auch akademische Gruppen und Firmen angeregt von den Erfordernissen der kommerziellen Nutzung des Internets größeres Geschick bei der Verschlüsselung. Offenbar versäumte die NSA, den Zugang zu diesen Systemen dauerhaft zu gewährleisten, indem sie amerikanische Firmen (wie Microsoft) und Verbündete überredete, mit ihnen zusammenzuarbeiten (durch die Hinterlegung von Chiffrierschlüsseln bzw. den Einbau bestimmter Chips). Andere Regierungen glaubten jedoch nicht, dass diese Maßnahmen lediglich zur Strafverfolgung bestimmt seien, und amerikanische Firmen befürchteten den Verlust ausländischer Märkte, wenn die entsprechenden Chips installiert würden. ${ }^{10}$ Trotzdem arbeiteten die Strafver-

US-Marine und US-Luftwaffe in den Jahren 1998/99, dass das ECHELON System noch zum Zeitpunkt tätig war als Enemy of the State in den Kinos anlief. (Vgl. www.zdnet.co.uk/ news/2000/25/ns-16204.html)

10 Es gibt einen grundlegenden Unterschied zwischen den Anforderungen der Gesetzgebung an das Abfangen von Nachrichten und denen, die an das Nachrichtenwesen gestellt werden. Strafverfolgungsbehörden fangen gewöhnlich eine bestimmte Leitung oder eine Gruppe von Leitungen $\mathrm{ab}$ und benötigen dafür eine richterliche Genehmigung. Nachrichtendienstliche Organe betreiben hingegen weit gestreute „Schleppnetz“-Aktivitäten. Sie agieren mit einer 
folgungsbehörden der Europäischen Union - weitgehend außerhalb parlamentarischer Kontrolle - mit dem FBI zusammen, um Geräte zum gesetzlich erlaubten Abhören von Telekommunikationsanbietern (z.B. Internetprovidern) zu entwickeln, was in den Vorschlag an 25 Staaten mündete, ein System namens ENFOPOL (Bernal 2001: 6) zu schaffen.

Parallel zu diesen Initiativen hat die US-Regierung ein sehr starkes und öffentlich bekundetes Interesse am Problem der Geldwäsche in Verbindung mit dem illegalen Drogenhandel gezeigt, dessen Umsätze in den USA 1991 auf mehr als 100 Mrd. Dollar geschätzt wurden. 1990 verarbeitete das Clearing House Interbank Payment System, das hauptsächlich für den Großhandel bestimmte elektronische Geldtransfersystem, rund 37 Millionen Überweisungen zwischen Banken in den USA und ausländischen Banken mit einem Wert von etwa 222 Billionen Dollar; obwohl also der absoluten Größe nach durchaus erheblich, macht Geldwäsche aus Drogengeschäften nur ein kleines Bruchstück aller Transaktionen aus (General Accounting Office 1991: 13). Ein großer Teil dieser Geldmittel wurde durch US-Banken und „Offshore“Banken gewaschen. Viel davon wird mit Hilfe elektronischer Geldtransfers gewaschen, aber trotz ihrer ausgefeilten Überwachungstechniken behaupten die US-Aufsichtsbehörden, dass das immer noch schwer zu verfolgen sei. Man könnte sich jedoch vorstellen, dass die NSA in der Lage wäre, ihnen bei dieser Aufgabe zu helfen.

Anfang der neunziger Jahre setzten die Vereinigten Staaten dann interne Prüfungskommissionen wie etwa FINCEN ein, die die Finanzbürokratien, Polizei- und Sicherheitsbehörden zur Koordinierung ihrer Strategie zusammenführten. Im Bemühen Geldwäsche zu unterbinden unterzeichnete FINCEN einen Vertrag mit dem Los Alamos National Laboratory, um ein statistisches Prüfgerät zu entwickeln, das in der Lage sein sollte, ,anormale Barguthaben bei Banken zu erkennen und auf potentiell illegale Aktivitäten hinzuweisen“. Der US-Zoll empfahl eine ganze Reihe anderer Maßnahmen einschließlich einer „Sperrcodierung“ der US-Währung, um „die Identifikation und Verfolgung von 'schmutzigem Geld' zu erleichtern, während es den Kreislauf der Geldwäsche durchwandert. (...) Das Gesetz zur Verbrechensbekämpfung (Crime Control Act) von 1990 weist das Finanzministerium an, diese Technologie zu erproben" (General Accounting Office 1991: 50)

In den neunziger Jahren führten die USA mit mindestens 50 ausländischen Regierungen eine ganze Serie von Verhandlungen über die Protokolle zur $\mathrm{Zu}-$ sammenarbeit und übten auf viele andere Druck aus, dem Ersuchen, Informationen zu erhalten, nachzugeben. Der G7-Gipfel von Paris im Juli 1989 setzte eine Financial Action Task Force ein, um Maßnahmen gegen Geldwä- 
sche zu fördern. Der Bericht dieser Arbeitsgruppe, der Definitionen von Geldwäsche und Empfehlungen zu ihrer Bekämpfung enthielt, wurde am 19. April 1990 veröffentlicht. Er wurde beim G7-Treffen in Houston 1990 bestätigt, und die Tätigkeit der Arbeitsgruppe wurde um ein weiteres Jahr verlängert. Die Administration hielt jedoch die tatsächlichen Inhalte und Ergebnisse dieser Verhandlungen - nicht nur mit den G7-Staaten, sondern auch mit der großen Zahl anderer Länder - unter Verschluß und hat auch viele ihrer Akten, die die Ausführung der US-Gesetzgebung betreffen, geheimgehalten, sogar vor dem General Accounting Office des Kongresses (General Accounting Office 1991: 52/3).

Gleichzeitig glauben die Kontinentaleuropäer den amerikanischen Behauptungen nicht, dass ECHELON nicht zur Wirtschaftsspionage benutzt worden sei. Angeführt von Frankreich, haben sie begonnen, konkurrierende Systeme zu installieren, was zum Teil aus wirtschaftlichen Gründen geschah, zum Teil zum Schutz der Privatsphäre, da die öffentliche Meinung in der Europäischen Union zunehmend darüber besorgt zu sein scheint, wie das amerikanische Überwachungswesen ihre internationalen und lokalen Telefongespräche, Faxe und e-mails abhören kann. Allerdings geben die „UKUSA“-Vereinbarungen den USA einen massiven Brückenkopf in Europa und die Fähigkeit, viel von den europäischen Telekommunikationsmitteln und Datenflüssen unter ständige Überwachung zu stellen. Dies wird möglicherweise durch die Liberalisierung von Dienstleistungen unter einem zukünftigen GATS-Regime noch erleichtert werden. Die (defensive) Verschlüsselung wird wohl zunehmen, da Berichte des Europa-Parlaments von 1998 - wie auch andere Quellen - zeigen, dass die USA auf nachrichtendienstlichem Wege geschäftliche, industrielle und finanzielle Informationen sammeln, um amerikanische Unternehmen und ihren weiteren ökonomischen Interessen zu nützen. ${ }^{11}$

\section{Ein Ausschnitt aus der dunklen Seite des finanziellen Panopticon: $\mathrm{BCCl}$}

Der letzte Abschnitt dieses Aufsatzes untersucht korrupte Aspekte der Beziehung zwischen dem internationalen System, der „Offshore-Welt und einer globalen Finanzordnung, die eine relativ freie Kapitalmobilität und Kapitalflucht zuläßt. Wie wir sehen werden, haben Geldwäsche, Betrug und Korruption in der Tat nicht nur mit finanziellen Kreisläufen, sondern auch mit den politischen Strukturen des modernen Kapitalismus zu tun.

Raymond Baker, Gastdozent für Wirtschaftswissenschaften an der USBrookings Institution und eine Autorität bei der Untersuchung illegaler Kapitalflucht, weist darauf hin, dass Korruption nur den wesentlich kleineren

11 Für weitere Informationen zu einem Verschlüsselungsdienst, der von einem Zusammenschluß mehrere privater Datenbanken entwickelt wurde, siehe www.protegrity.com 
Teil solcher Bewegungen ausmacht, obwohl sie sich in den späten neunziger Jahren auf stolze $20 \mathrm{Mrd}$. Dollar jährlich belief. ${ }^{12}$ Vielmehr stellt das, was Baker die „Verfälschung der Handelspreise“ durch transnationale Konzerne und ihre Tochtergesellschaften nennt, bei weitem die Masse des Problems der Kapitalflucht dar. Hier kooperieren Käufer und Verkäufer bei der illegalen und geheimen Bewegung von Geldern, die auf mindestens $80 \mathrm{Mrd}$. Dollar jährlich geschätzt werden. Diese Transfers werden von Netzwerken privater Firmen in Entwicklungsländern, postkommunistischen Staaten, den Vereinigten Staaten und in Europa durchgeführt. Insgesamt sind es also rund 100 Milliarden Dollar jährlich, die der Westen allein auf diesen beiden Wegen aus den Volkswirtschaften der Übergangs- und Entwicklungsländer herausschafft (Baker 1999). Etwa die Hälfte davon, 50 Milliarden Dollar jährlich, fließt in die USA.

Baker fügt hinzu, dass diese 100 Milliarden Dollar, die jährlich als illegales Fluchtkapital in den Westen gelangen, „den Deckmantel für einen noch viel größeren Betrag von kriminellem Geld abgeben, das im Westen gewaschen wird. Schätzungen reichen von 500 Milliarden bis zu einer Billion Dollar jährlich.“ Die zwei Typen von Kapitalzuflüssen, kriminelle Geldwäsche und illegales Fluchtkapital, durchlaufen dieselben Mechanismen des internationalen Finanzsystems und landen in „westlichen Kassen“. „Das (US-) Finanzministerium schätzte mir gegenüber, dass $99,9 \%$ des schmutzigen Geldes, das in den USA zur Wäsche angeboten wird, erfolgreich auf sicheren Konten deponiert ist“ (Baker 1999). Baker führt weiter aus:

„Der Gesamtbetrag ausländischer Hilfe von den USA, den OECD-Staaten und der Weltbank, die in die Entwicklungs- und Übergangswirtschaften geht, beläuft sich auf etwa $50 \mathrm{Mrd}$. Dollar im Jahr. Die 50 Mrd., die jährlich hereinkommen, werden vollkommen aufgewogen durch die 100 Mrd., die mit westlicher Unterstützung illegal wieder hinaus- und zurückfließen. Das Bild, das wir - als Amerikaner, Europäer oder Japaner - in unseren Köpfen haben, ist das der Großzügigkeit. Es gibt vor, wir würden großzügig Geld an weniger glückliche Länder rund um die Welt verteilen. Das zutreffendere Bild ist, daß wir für jeden Dollar, den wir über dem Tisch überreichen, zwei oder mehr Dollar unter dem Tisch zurücknehmen" (Baker 1999).

Diese Aspekte des globalen Finanzsystems kamen im Fall der jetzt bankrotten BCCI zum Vorschein. Diese „Bank“ wurde von der CIA auf verschiedene Weise benutzt, vor allem um Gelder zur Finanzierung der geheimen Kriege in Afghanistan und im Iran einzuschleusen, insbesondere zu der Zeit, als William Casey CIA-Direktor war (1981-87). Der Financial Times zufolge operierte die in Luxemburg registrierte BCCI in 73 Ländern. Die BCCI präsentierte sich so, als ob sie von Ländern der Dritten Welt, insbesondere Abu Dhabi und Pakistan, betrieben würde und sich in deren Besitz befände. Sie war bekannt dafür, dass sie im Hinblick auf ihre Tätigkeit, ihren Charakter und ihre Leitung mit der Dritten Welt verbunden war. In Wirklichkeit war sie eine

12 Die legale Komponente von Fluchtkapital, d.h. das Kapital, das nach Steuerzahlung ins Ausland fließt, da die Investoren kein Vertrauen in die entsprechende Ökonomie mehr haben, bleibt hier unberücksichtigt. 
supranationale Bank, die durch viele geheime „Offshore“-Komponenten gekennzeichnet war, ohne einen einzelnen Heimatstaat zu besitzen. Dadurch war sie von einigen Beschränkungen durch regierungsamtliche Aufsicht und Vorsichtsmaßregeln befreit und in der Lage, sich in umfangreicher Geldwäsche und einer Reihe anderer illegaler Aktivitäten zu engagieren, und dabei Anleger in der ganzen Welt, vor allem aber in Ländern der Dritten Welt, auszuplündern.

Der wesentliche Punkt, den ich hier hervorheben möchte, ist, dass die Bedeutung dieser Konstellation nicht ausschließlich die BCCI betrifft, denn sie ist eng mit den vielfältigen Problemen der Steuerhinterziehung und Kapitalflucht verbunden, die mit der „Offshore“-Welt zu tun haben und zu den Hauptmechanismen wurden, mit denen die Vermögenswerte der weniger entwickelten Länder in der oben beschriebenen Weise abgezogen werden. Ein großer Teil dieses Wohlstands fließt schließlich in die Kassen der westlichen Banken, ohne die eine Einrichtung wie die BCCI nicht existieren könnte.

Aber wir greifen der Geschichte vor. Lassen Sie uns mit William Casey beginnen. Casey war ein OSS-Veteran, der von 1943 bis 1945 Spionagenetze in Deutschland leitete; später gehörte er dem Beratungsgremium der Behörde für Rüstungskontrolle und Abrüstung an. Er war aber auch ein erfolgreicher und umtriebiger Geschäftsmann, war in den frühen siebziger Jahren Vorsitzender der US-Kommission für Wertpapiere und Devisen (US Securities and Exchange Commission) und schließlich war er auch Vorstand der USamerikanischen Export-Import Bank, was ihm, wie Bob Woodward in Veil bemerkte, Zutritt zum „Klub der Zentralbankchefs“ verschaffte. Casey wurde von Präsident Reagan an die Spitze der CIA berufen, um die Effektivität des US-Nachrichtendienstes nach den Mißerfolgen, die mit der Revolution im Iran verbunden waren, zu verbessern. Man glaubte, dass er die operative, geschäftliche und finanzielle Erfahrung mitbrachte, um die CIA in die neue konservative Ära zu führen und den Feind zurückzudrängen. Für Casey schloß das ein, die Koordination zwischen CIA und NSA zu verbessern und einen von den „HYPs“ (Absolventen der Universitäten von Harvard, Yale und Princeton) dominierten Nachrichtendienst gründlich umzustrukturieren. Laut Bob Woodward war Casey der führende Kopf hinter der geheimen Kriegführung, die die Reagan-Administration in den achtziger Jahren betrieb. Es ist behauptet worden, dass sich die Kosten dieser geheimen Operationen der CIA und „befreundeter“ Regierungen in den achtziger Jahren auf über 2 Mrd. Dollar beliefen und dass der Löwenanteil davon von korrupten pakistanischen Beamten bei der BCCI gestohlen worden sei (vgl. Kerry/Brown 1992a, im folgenden auch als Kerry-Report bezeichnet). Casey traf Hasan Agha Abedi, den Gründer der BCCI, „alle drei oder vier Monate" (Colhoun 1993: 40). Die BCCI wurde offenkundig in der Iran-Contra-Affäre benutzt, um - im Widerspruch zu US-Vorschriften und angesichts der Opposition des 
Kongresses - Waffenverkäufe der USA an den Iran und die nicaraguanischen Contras zu finanzieren (Colhoun 1993: 40/41). Mindestens 50 Millionen Dollar trieb die CIA direkt bei anderen (unbekannten) Regierungen für die Iran-Contra-Angelegenheit auf. Eine Schenkung von 10 Millionen Dollar, anscheinend von Brunei, erreichte wegen eines Schreibfehlers nie das Schweizer Bankkonto, für das sie bestimmt war (Cohen/Mitchell 1988: xii-xx).

Die BCCI brach mit wahrscheinlich über $10 \mathrm{Mrd}$. Dollar an verlorenen oder gestohlenen Guthaben in mehr als 70 verschiedenen Ländern zusammen, die Bank von England hatte ihre Geschäfte 1991 schließlich beendet. In den neunziger Jahren gelang es der Bank von England unter Ausnutzung ihrer Verbindungen zum britischen Establishment, Kritik an ihrer Inkompetenz und Mißwirtschaft und, wichtiger noch, eine Untersuchung nach US-Art abzuwehren; was stattfand, war die Bingham-Untersuchung, die zurückhaltend zu Werke ging und nicht verlangte, das Versagen der Bank von England nach Standards der internationalen Bankenaufsicht zu behandeln, wie sie mit der Bank für Internationalen Zahlungsausgleich vereinbart waren. Das geschah, obwohl es klare Beweise für die nachlässige und laxe Überprüfung einer Bank gab, die bis zu einem Wert von $20 \mathrm{Mrd}$. Dollar expandierte (Financial Times 1992). Die Bank von England wußte schon eine ganze Weile von der Mißwirtschaft bei der BCCI (mindestens von 1982 an und noch deutlicher seit 1988, als ernsthafte und konkrete Klagen über die BCCI bei der Bank von England eingingen und sieben BCCI-Mitarbeiter in Tampa, Florida, des Drogenhandels und der Geldwäsche überführt wurden). Auch Price Waterhouse die Rechnungsprüfer der BCCI in Großbritannien - gelang es zu dieser Zeit, einer gründlichen Nachforschung zu entgehen.

Viele politische Schlüsselfiguren waren weltweit in die illegalen Aktivitäten der BCCI verwickelt, darunter führende Gestalten des US-Establishments wie der frühere Verteidigungsminister Clark Clifford oder auch der ehemalige britische Premierminister Lord Callaghan, der mindestens sieben Jahre lang ein enger Freund von Hasan Agha Abedi gewesen war. Callaghan fungierte als inoffizieller Berater der BCCI.

Die meisten Opfer des BCCI-Skandals waren kleine Geschäftsleute in über 70 Ländern in der ganzen Welt. Eine Million kleine Kontoinhaber verloren ihr Geld, ebenso wie Zentralbanken, Regierungsorganisationen, Investmentfonds und Beamte (Kerry-Report 1992a: 99). Zu den betroffenen Ländern gehörten mehrere lateinamerikanische und karibische Staaten sowie Abu Dhabi, Bangladesh, Belgien, der Iran, Italien, Ungarn, Korea, Liberia, Libyen, Nigeria, Pakistan, die Philippinen, Saudi Arabien, Senegal, Spanien, die Schweiz, Thailand, die Türkei, die Vereinigten Arabischen Emirate, Jugoslawien und Sambia; in jedem dieser Länder gibt es Spuren von Bestechung und Korruption durch die BCCI. Diese hatte sich selbst als „Bank der Dritten Welt“ dargestellt: eine Bank, die den Unterprivilegierten der Welt dabei helfen würde, 
Zugang zum Finanzwesen unter Bedingungen zu bekommen, die sich normale Leute leisten könnten. Viele Beweise über die BCCI und ihre Verbindungen wurden im Kerry-Report offengelegt:

„Der Untersuchungsausschuß hat in Dokumenten und Zeugenaussagen umfangreiche Beweise dafür vorgelegt, daß sich die BCCI systematisch auf Beziehungen zu und, wenn nötig, Zahlungen an prominente politische Persönlichkeiten in den meisten der 73 Länder stützte, in denen sie tätig war. Sowohl Akten der BCCI als auch Aussagen ihrer früheren Mitarbeiter belegen, daß sich die Bank systematisch in den Besitz von Zentralbankeinlagen von Ländern der Dritten Welt brachte, daß sie politisches Personal materiell begünstigte und sich auf diese Personen verließ, wenn sie selbst Begünstigungen benötigte“ (Kerry/Brown 1992a: 101).

Die wichtigsten Ergebnisse des Kerry-Reports waren: dass die BCCI „internationale Finanzverbrechen in einem riesigen und globalen Maßstab begangen hat“; dass sie „bedeutende Führer und politische Persönlichkeiten überall in der Welt systematisch bestochen hat" (z.B. in Lateinamerika: die Präsidenten von Brasilien, Argentinien und Peru - Sarney, Menem und Garcia - waren alle mit der BCCI verquickt). Vielleicht noch dreister entwickelte die BCCI eine erfolgreiche Strategie, um die Banksysteme der USA und anderer Staaten zu infiltrieren, obwohl Regulierungsbarrieren dazu bestimmt waren, sie fernzuhalten. Das US-Justizministerium ließ Untersuchungen und Verfolgung schleifen; vielmehr war es der New Yorker Bezirksstaatsanwalt Morgenthau, der den Fall übernahm und damit indirekt die weltweite Schließung der BCCI herbeiführte. Die Buchprüfer der Bank „versäumten es, unschuldige Einzahler und Kreditgeber zu schützen“, trotz der Probleme, die die Revisoren seit Jahren kannten. Die CIA kannte und benutzte die BCCI, unterließ es aber, ihre Informationen weiterzugeben oder aufzudecken, was sie tat.

Fehlerhafte Entscheidungen von Beamten der US-Bankenaufsicht erlaubten es der BCCI, amerikanische Banken „heimlich zu übernehmen“, dies z.T., weil sie Anwälte mit guten Verbindungen hatte; so waren etwa Clark Clifford und Robert Altman Schlüsselfiguren, die für die BCCI in den Vereinigten Staaten tätig waren. Die Kontrolle der BCCI durch die Bank von England war „vollkommen unzureichend“, um die Einleger und Gläubiger der BCCI zu schützen, sie „hielt Informationen“ über massive Betrügereien 15 Monate lang „zurück“, bevor sie die Bank schloß. Die BCCI benutzte politischen Einfluß ebenso wie Öffentlichkeitsarbeit als Teil ihrer Taktik, „nahm aktiv die Freundschaft führender politischer Figuren der USA in Anspruch und leistete Zahlungen an diese Personen". Capcom, die für den Warenhandel zuständige Tochtergesellschaft der BCCI, betrieb in den USA weitgehend anonyme Handelsgeschäfte im Umfang von mehreren Milliarden Dollar, was in einem erheblichen Grad Geldwäsche einschloß. „Gleichzeitig nahm Capcom bedeutsame Verbindungen $\mathrm{zu}$ wichtigen Akteuren der US-Telekommunikationsindustrie und ausländischen Geheimdienstleuten auf (Kerry/Brown 1992b).

Während es immer noch unmöglich ist, alles über die mysteriösen Beziehungen zwischen der BCCI, dem amerikanischen Nachrichtendienst und den 
Behörden, die für die Steuerung des amerikanischen Finanzwesens verantwortlich sind, zu wissen, macht der Kerry-Report deutlich, dass die CIA sowohl die BCCI als auch die First American Bank, ihre im Geheimbesitz gehaltene US-Tochter, auch dann noch weiterhin für ihre Operationen benutzte, als sie längst wußte, dass die BCCI ein ,fundamental korruptes und kriminelles Unternehmen“ war. Darüber hinaus hält der Kerry-Report fest:

„Der durchdringende Charakter der Korruption von Amtspersonen durch die BCCI in so vielen Ländern ruft auch weitergehende Fragen hinsichtlich des Beharrungsvermögens von Korruption als eine im Geschäftsleben überall in der Welt allgemein übliche Vorgehensweise hervor. Mitarbeiter der BCCI behaupten, daß deren Praktiken typisch waren für die, die auch andere Banken (einschließlich US-Banken) anwandten, die in Entwicklungsländern Geschäfte machen. Wenn das wahr wäre, würde das z.B. bedeuten, daß internationale Institutionen wie der IWF und die Weltbank, die von den USSteuerzahlern finanziert werden, durch das heimliche Einverständnis zwischen den Regierungen dieser Länder und unethischen Banken laufend betrogen werden“ (Kerry-Report 1992b).

Wie schon erwähnt, waren die Hauptwege für diese Art von Betrug die „Offshore“-Strukturen zur illegalen Aneignung von Kapitalfonds in Verbindung mit Geldwäsche. Ein neuerer Bericht der Vereinten Nationen schätzte den Umfang der Guthaben, die sich derzeit auf schwarzen Konten befinden, auf rund acht Billionen Dollar. Es gibt auch etwa drei Millionen weitgehend anonyme Briefkastenfirmen und illegal operierende Trusts, die lediglich dazu dienen, Eigentumsverhältnisse zu verbergen (Blum 1999'13). Daher ist es außerordentlich schwierig, Informationen über Steuerflucht, Finanzbetrug, Geldwäsche und andere transnationale kriminelle Aktivitäten zu erhalten.

Ein anderer Grund hierfür ist, dass die verschiedenen Teile des Staatsapparates unterschiedliche Interessen im Hinblick auf die Regulierung des Finanzwesens haben. So war die CIA, wie Kerry häufig bemerkt, alles andere als kooperativ gegenüber den Untersuchungen des Kongreß. So stellte Kerry „ungewöhnliche" Aspekte in der Art und Weise fest, wie die CIA 1985 und 1986 dem US-amerikanischen Finanzministerium darüber berichtete, was sie über die BCCI wußte und welche Verbindungen sie zu ihr hatte, als letztere versuchte, die Kontrolle über die größte Bank in Washington DC, die First American, zu bekommen. Tatsächlich unterließ es die CIA 1986, die beiden Behörden zu informieren, die eine gesetzliche Verpflichtung hatten, Untersuchungen einzuleiten: die amerikanische Zentralbank und das Justizministerium. Ebenso wurden Originalberichte und Beweisstücke von der CIA als verloren bezeichnet, so dass Kerry schließlich einen „rekonstruierten“ Bericht bekam und die CIA-Mitarbeiter, die mit den Originalberichten beauftragt gewesen waren, unter Eid verneinten, jemals entsprechende Informationen erhalten zu haben. ${ }^{14}$ Der Kerry-Report läßt deutlich werden, warum nicht je-

13 Blum ist Partner bei Lobel, Novins \& Lamont, sowie Co-Autor der UN-Publikation Financial Havens, Banking Secrecy and Money Laundering.

14 Am 19. Februar 1992 sagte unter anderem Douglas P. Mulholland aus, damals Leiter des Nachrichtendienstes im Finanzministerium. Als erfolgreicher CIA Beamter von William Ca- 
der im Nachrichtenapparat der USA davon begeistert sein konnte, dass allzu viel enthüllt würde:

„Frühere CIA-Mitarbeiter, darunter der ehemalige CIA-Direktor Richard Helms und sein Nachfolger William Casey, ehemalige und aktive Mitarbeiter ausländischer Geheimdienste wie Kamal Adham und Abdul Raouf Khalil sowie wichtige ausländische Agenten der USA wie Adnan Kashoggi und Manucher Ghorbanifar gehen in den kritischen Phasen der Geschichte der BCCI bei dieser ein und aus und nehmen gleichzeitig an der Gestaltung von Schlüsselereignissen der USAußenpolitik teil, von den Friedensverhandlungen von Camp David bis zur Bewaffnung des Iran als Teil der Iran-Contra-Affäre. Aber die CIA hat fortgesetzt behauptet, daß sie keine Informationen hinsichtlich einer Beteiligung dieser Personen habe, was die Frage aufwirft, welche Qualität die Nachrichten haben, die die CIA im allgemeinen bekommt, oder wie aufrichtig sie gegenüber dem Untersuchungsausschuß ist. Die Beteuerungen der CIA, über die jeweilige Rolle jener Personen in der BCCI überhaupt nichts zu wissen, sind unvereinbar mit der frühen Kenntnis der Behörde von vielen kritischen Aspekten der Operationen, der Struktur, des Personals und der Geschichte der Bank“ (Kerry/Brown 1992b).

Man könnte noch viele andere gute Gründe für das Schweigen seitens der CIA finden. Die BCCI kultivierte Verbindungen zu wichtigen politischen Personen in den USA und im Ausland. So erfuhr die BCCI z.B. in Washington DC professionelle Unterstützung nicht nur, wie wir festgestellt haben, von Clark Clifford und Altman, sondern auch durch frühere Senatoren und Kongreßabgeordnete (John Culver, Mike Barnes), frühere Bundesstaatsanwälte, einen ehemaligen Beamten des Außenministeriums, einen früheren Berater des Weißen Hauses und ehemaligen Bevollmächtigten der Zentralbank. Die BCCI nahm auch die Hilfe von Henry Kissinger in Anspruch, der sich entschied, mit dieser Bank keine Geschäfte zu machen, sie aber seinen eigenen Anwälten empfahl. In dieser Beziehung verheimlichten Clifford und Altman viel von dem, was sie wußten, gegenüber der US-Bankenaufsicht und dem Kongreß. Ebenso „entwickelte die BCCI systematisch Freundschaften und Kontakte zu bedeutenden politischen Gestalten der USA. Darunter befinden sich der frühere Präsident Jimmy Carter, Jesse Jackson, Andrew Young u.a. Die Zahlungen, die die BCCI an Andrew Young leistete, als dieser noch im Staatsdienst tätig war, waren zumindest ungewöhnlich und - allem Anschein nach - unzulässig“ (Kerry/Brown 1992b).

Eine wichtige Person in dieser Affäre war Kamal Adham. Von Kerry als „Pate der BCCI in den nahöstlichen Geheimdiensten“ bezeichnet, war er der wichtigste Verbindungsmann der CIA für den ganzen Nahen Osten von Mitte der sechziger Jahre bis 1979 (Woodward 1987: 352). Adham erreichte eine Übereinkunft mit dem Bezirksbevollmächtigten von New York, in der seine Rolle

sey im Finanzministerium eingesetzt, zog sich Mulholland 1987 aus der CIA zurück, um für den Bush-Wahlkampf tätig zu sein, bevor er zum Leiter des Nachrichtendienstes im amerikanischen Außenministerium ernannt wurde. Als Mulholland 1985 den CIA Bericht dem Finanzminister Donald Regan vorlegte, bezeichnete er ihn als „Dynamit“, da die BCCI, trotz eines Verbots versuchte, die Kontrolle über die größte Bankholding im Washingtoner Stadtgebiet zu erlangen. In der Tat war bereits die Washington Post und die Finanzpresse auf die BCCI aufmerksam geworden. 
als BCCI-Repräsentant in den USA anerkannt wurde. Als die BCCI 1972 gegründet wurde, war Adham Schwager von König Faisal von Saudi-Arabien und Chef des saudischen Geheimdienstes. Wie Bob Woodward bemerkte, war der „legendäre und ungeheuer reiche“ Adham ein Mann mit „ungewöhnlich engen" Beziehungen zur CIA. Adham fungierte als der Hauptverbindungsmann der Saudis und der CIA zum damaligen ägyptischen Vizepräsidenten Sadat (der offenbar, als er finanzielle Schwierigkeiten hatte, von der CIA mit einem regulären Einkommen versorgt wurde). „Es war unmöglich festzustellen, wo in diesen Arrangements saudische Interessen endeten und amerikanische CIA-Interessen begannen" (Woodward 1987: 352). Adham wurde offiziell zum „Hauptinvestor“ bei der Übernahme der First American, indem er als Strohmann der BCCI auftrat. Obwohl er in Anhörungen der Zentralbank erschien, wo er Auskunft über sein Vermögen und seinen Werdegang gab und behauptete, nur ein passiver Investor zu sein, unterließ es Adham, etwas über seinen nachrichtendienstlichen Hintergrund und seine Beziehungen zur CIA verlauten zu lassen, oder auch darüber, dass er wichtige Kontakte zwischen den USA und „seinem langjährigen Freund, Anwar el Sadat geknüpft hatte, als es darum ging, das Camp David-Abkommen auszuhandeln" (Kerry/Brown 1992b).

Offenkundig war es das Ziel der BCCI, Einlagen, Vermögenswerte und Profite zu maximieren, und gewiß können wir vermuten, dass ein großer Teil von den $10 \mathrm{Mrd}$. Dollar, die verloren gingen, auf schwarze Konten transferiert gewaschen und in sichere „legale“ Häfen zurückgebracht wurde. Ein großer Teil der geographischen Expansion der Bank wurde durch Fusionen und Übernahmen betrieben. In Lateinamerika pflegten die Übernahmespezialisten der BCCI den Kontakt zu führenden Leuten der Zentralbanken und zu Regierungsbeamten in Argentinien, Brasilien, Kolumbien, Peru, Venezuela und anderen Ländern, um „für den Kauf geeignete Banken“ zu finden. Manchmal halfen dabei auch Mitarbeiter internationaler Finanzinstitutionen wie dem IWF. Im allgemeinen gab es in den lateinamerikanischen Staaten deutliche „Beschränkungen für die Möglichkeit ausländischer Banken, einheimische Banken zu kaufen" oder die Kontrolle über die Kapitalmehrheit zu erlangen. ${ }^{15}$ Deshalb machte die BCCI „in jedem Land prominente Persönlichkeiten (aus), die bereit wären, beim Erwerb lokaler Institute als Strohmänner aufzutreten, ohne dass dies ein Risiko für sie bedeutete, während die Bank ihre Käufe heimlich finanzieren würde, genau wie sie es in den Vereinigten Staaten getan hatte" (Kerry/Brown 1992b) Die BCCI leistete verschiedene Zahlungen an Personen, die Übernahmen erleichterten, Schmiergelder für Beamte eingeschlossen, und obwohl sich die Details jeder Transaktion unterschieden,

15 Dies betrifft die Zeit vor der weitergehenden Liberalisierung und dem Schutz der Rechte ausländischer Investoren durch WTO, NAFTA und andere bilaterale Verträge. 
war das Muster einige Jahre früher vorgezeichnet worden, als die Bank ihre geheime Übernahme der First American durchführte. In Brasilien z.B. „belohnte“ die BCCI einige der angesehensten Diplomaten und Politiker des Landes, damit sie ihr dabei halfen, die Gesetze ihres eigenen Landes zu umgehen.

Ein anderer Sachverhalt, der es erlaubt, die Beziehungen der BCCI zu ausländischen Regierungen einzuschätzen, waren die Einlagen, die sie von vielen Zentralbanken erhielt. In gewisser Hinsicht könnte es logisch erscheinen, wenn sich die Zentralbank eines Entwicklungslandes entschied, ihr Geld bei der BCCI anzulegen, galt diese doch als „Bank der Dritten Welt“, die ihren Kunden die besten Dienstleistungen bot. Zentralbankleute wußten jedoch, dass die BCCI, da sie de facto ein Komplex von Dachgesellschaften war, keinen letztlich haftenden Geldgeber und auch keine fundierte Rechnungsprüfung hatte. Daher hätte jeder Zentralbankchef, der etwas auf sich hielt, wissen müssen, dass jegliche Einzahlungen bei der BCCI sehr riskant waren: wenn diese bankrott ginge, wären die Zentralbankfonds nicht geschützt. Nichtsdestotrotz wurden die Reserven von rund 20 Zentralbanken auf unsicheren Konten bei der Bank angelegt, in manchen Fällen in einem nennenswerten und sehr unvorsichtigen Umfang.

Was schließlich die Aufsichtspflichten von Zentralbanken betrifft, so wurde im März 2001 angekündigt, dass die Bank von England, die normalerweise von Strafverfolgung verschont bleibt, nun zum ersten Mal überhaupt einem Prozeß entgegensieht, den Deloitte \& Touche, die Konkursverwalter der BCCI, angestrengt haben. Diese fordern 550 Millionen Pfund als Schadensersatz für BCCI-Einleger; mit Zinsen könnte sich das auf eine Milliarde Pfund summieren. Das Oberhaus stimmte mit absoluter Mehrheit für die Zulassung der Klage gegen die Bank von England wegen vorsätzliche Vernachlässigung der Aufsichtspflichten. Die Liquidatoren haben seit 1993 versucht, die Bank zu verklagen. Trotzdem ließ die Bank von England verlauten, der Parlamentsbeschluß sage nichts über ein Fehlverhalten oder die wesentlichen Punkte der Klageschrift aus und man habe „völliges Vertrauen“, den Prozeß (der voraussichtlich 2002 beginnen wird) zu gewinnen (Croft 2001).

\section{Schlussfolgerungen}

Wie in diesem Aufsatz gezeigt wurde, ist die Suche nach einem globalen Panopticon im Finanzwesen ein modernistischer Mythos, der ständig von den Widersprüchen der Globalisierung unterminiert wird. Diese Widersprüche setzen jedem Projekt globaler Überwachung unter der Herrschaft des Kapitals finanzielle oder politische Grenzen. $\mathrm{Zu}$ diesen Widersprüchen zählen nicht nur jene zwischen Arbeit und Kapital, sondern auch die Beziehungen zwischen herrschenden kapitalistischen Staaten. Oberflächlich gesehen scheint es so, dass die Regierungen der großen kapitalistischen Staaten ein gewisses In- 
teresse an Überwachung haben, die auf vorsichtiger Regulierung basiert. Vielleicht hat auch eine Mehrheit dieser Staaten Interesse an einer Überwachung der Liberalisierung in der früheren Sowjetunion und der Dritten Welt - bis zu einem gewissen Grad. Jedoch gibt es in einer von neuen Wettbewerbsideologien dominierten Welt auch Reibungsverluste zwischen den führenden Kräften in Kontinentaleuropa und in Nordamerika bei der Diskussion über Regulierung und nationale Sicherheit. Dies wird deutlich, wenn wir etwa die Auseinandersetzungen über die Regulierung der Kapitalmärkte betrachten. Es sind Auseinandersetzungen, die bis zu den Klassenstrukturen und den politischen Formen beider Systeme zurückverfolgt werden können und die häufig als Rivalität zwischen der angelsächsischen Form des „Shareholder“Kapitalismus und der kontinental-europäischen „Stakeholder“-Gesellschaft (Formen einer sozialen Marktwirtschaft) beschrieben werden, obwohl es auch Anzeichen für den zunehmenden Einfluß des Shareholder-Kapitalismus auf dem Kontinent gibt. Letztlich brauchen westeuropäische Staaten irgendeine Form von Protektion, um dem intensivierten Eindringen und der Überwachung durch den amerikanischen Nachrichtenapparat entgegentreten zu können. Dies wird in der scharfen transatlantischen Debatte zu ECHELON deutlich.

Natürlich ist das noch nicht alles. In der Tat gibt es selbst unter den Hauptverbündeten der USA nicht nur völlige Harmonie und reibungslose Kooperation. Als der Kerry-Report veröffentlicht wurde, waren seine Untersuchungen unvollständig, was im wesentlichen daran lag, dass die Regierungen von Großbritannien und Abu Dhabi die Zusammenarbeit verweigerten. Der Senatsausschuss, an den der Kerry-Report ging, empfahl abschließend eine ,aggressive Vorgehensweise“, die innerhalb der Regierung der Vereinigten Staaten und zwischen dieser und den Regierungen anderer Staaten koordiniert werden sollte, um internationale Finanzverbrechen zu bekämpfen. Er rief zu Maßnahmen gegen ausländischen Datenschutz und Vertraulichkeitsbestimmungen auf, die Kriminelle schützen. Seine Untersuchungen hatten unterschiedliche Umgangsweisen mit Überwachung und Regulierung innerhalb des US-amerikanischen Staatsapparates enthüllt. Daher forderte Kerry zusätzliche Aufsichtsmechanismen, um die Verantwortlichkeit der CIA und anderer Institutionen, früherer und jetziger Regierungsbeamter und privater Rechtsanwälte in Bezug auf die Offenlegung von Informationen und zwingenden Verhaltensregeln sicherzustellen. Kerry forderte auch verstärkte Maßnahmen gegen Geldwäsche sowie eine stärkere Regulierung von in- und ausländischen Finanzunternehmen, die in den USA operieren, inklusive eines strengen Verbots des Gebrauchs von Strohmännern zur Kontrolle von Unternehmen.

Viele dieser Forderungen wurden nicht umgesetzt, was man mit den oben erwähnten Konflikten zwischen den verschiedenen staatlichen Instanzen wie auch mit den Interessen der oft anonym bleibenden Kapitalbesitzer erklären kann: Viele von ihnen nutzen die Offshore-Ökonomie, um ihre eigenen Inte- 
ressen zu verfolgen. In einer Welt des mobilen Kapitals sind die Angebote der Offshore-Ökonomie ständig präsent. In der Tat werden alle Entwicklungspläne der einzelnen Staaten durch die globale Finanzwelt diszipliniert allerdings sind einige disziplinierter als andere. Wie gezeigt wurde, sind die USA in Finanzangelegenheiten die berühmte Ausnahme von der Regel. Besser als jede andere Regierung sind sie in der Lage, aus den Risiken und Möglichkeiten, die die neuen Finanzstrukturen bieten, Kapital zu schlagen. Jedoch gibt es keinen Anlass, daraus zu schließen, dass Staatsapparate gänzlich die Fähigkeit verloren hätten, die Finanzwirtschaft zu regulieren, geschweige den mobiles Kapital nicht mehr unter Kontrolle haben. Das Einfrieren von iranischen Guthaben im Wert von 14 Mrd. Dollar nach der Revolution von 1979 dient als Beispiel für die einzigartige Fähigkeit der USA, in das internationale Bankensystem einzugreifen und ein gewisses Maß direkter Kontrolle auszuüben. Die nationalen Sicherheitsinteressen der Vereinigten Staaten sind sicher noch mächtig genug, um „bestimmte“ Interessen von Bankern zu überwinden; wobei sich die Frage stellt, ob dies auch noch zu Beginn des 21. Jahrhunderts der Fall ist (Alerassool 1993: 30/1). Es steht außer Frage, dass die USA (und ihre G7-Partner) das globale Finanzwesen in einer Weise lenken könnten, die zum Beispiel den Kapitalfluß vom Rest der Welt in den Westen zum Erliegen brächte - wenn sie dies wollten.

An dieser Stelle sollten wir vielleicht pessimistisch sein. Der Kerry-Report hat gezeigt, wie wenig adäquat Führungsstrukturen in der globalen politischen Ökonomie in vielerlei Hinsicht sind: sie sind unverantwortlich in sozialer Hinsicht und nur unzureichend demokratisch kontrolliert. Von besonderem Interesse sind die zunehmend „unabhängigen“ Zentralbanken, die heute mehr denn je gegen demokratische Überprüfungen geschützt sind. Zudem zeigt die Erfahrung mit der BCCI (und einer Reihe anderer Fälle), dass der gegenwärtige Kapitalismus nur eingeschränkt den Gesetzen unterliegt und darüber hinaus mit doppeltem Maßstäben operiert. Viele der Herrschenden favorisieren - aus Überzeugung oder aus der Situation heraus - eine Zwangsherrschaft, die auf Dominanz und Korruption, Betrug und Einschüchterung basiert. Dieser doppelte Standard unterminiert jeden Anspruch auf Legitimität, vor allem im Kontext der zunehmenden Finanz- und Wirtschaftskrisen in den letzten beiden Jahrzehnten, von denen viele mit Kapitalflucht und Glaubwürdigkeitsproblemen von Regierungen in Zusammenhang stehen.

Übersetzung aus dem Englischen: Astrid von Enzberg

\section{Literatur}

Alerassool, Mahvash (1993): Freezing Assets: The USA and the Most Effective Economic Sanction New York.

Baker, Raymond (1999): Remarks at „Criminal Money Laundering and Illegal Flight Capital“, in: Brookings Press Briefing vom 29. September. 
Bauman, Zygmunt (1998): Globalization: The Human Consequences. New York.

Bentham, Jeremy (1995): The Panopticon Writings. London/New York.

Bernal, Javier (2001): Big Brother is Online: Public and Private Security in the Internet 2001 unter http://derecho.org/comunidad/echelon/25.3.2001.

Blum, Jack (1999): Remarks made at „Criminal Money Laundering and Illegal Flight Capital“, Brookings Institution Press Briefing vom 29.September.

Blum, Jack et al. (1999): Financial Havens, Baning Secrecy and Money Laundering. New York.

Braithwaite, John und Peter Drahos (2000): Global Business Regulation, Cambridge.

Bronskill, Jim (2001): Canada a Key Snooper in Huge Spy Network, in: Ottowa Citizen vom 25.März.

Camdessus, Michel (1999): Capital Flows, Crises, and the Private Sector, Washington DC.

Cohen, William S. und George J. Mitchell (1988): Men of Zeal: A Candid Inside Story of the IranContra Hearings, New York.

Colhoun, Jack (1993): BCCI: Bank of the CIA, in: Covert Action, Vol.44.

Croft, Jane (2001): BCCI Liquidators can Sue BoE, in: Financial Times vom 22. März.

Davies, Simon G. (1993): The Zones of Surveillance: a Methodology to Quantify and Score Surveillance, in: New Technology, Surveillance and Social Control, Queen's University, Kingston, Ontario, $10-11$.

Drew, Christopher (2001): Listening, Looking: Old Methods Still Work, in: New York Times vom 14. April, A5.

Europa-Parlament (1999): Development of Surveillance Technology and Risk of Abuse of Economic Information, STOA Vol. 5, Luxemburg.

Financial Times (1992): Financial Regulation Report, November.

Fitchett, Joseph (2001): Spying from Space: U.S. to Sharpen the Focus, in: International Herald Tribune vom 10. April, 1.

Foucault, Michel (1993): Space, Power and Knowledge, in: The Cultural Studies Reader, hrsg. von S. During, London.

Gill, Stephen (1995): The Global Panopticon? The Neo-Liberal State, Economic Life and Democratic Surveillance, in: Alternatives, Vol. 20, Nr.1, 1-49.

- (1998). European Governance \& New Consitutionalism: EMU \& Alternatives to Disciplinary Neo-Liberalism in Europe, in New Political Economy, Vol.3, Nr. 1, 5-26.

Kerry, John und Hank Brown (1992a): The BCCI Affair. A Report to the Senate Committee on Foreign Relations by the Subcommittee on Terrorism, Narcotics and International Operations. 102. Kongress, 2. Sitzung, Washington DC, US Government Printing Office. (Gedruckte Version des Kerry-Reports)

- (1992b): BCCI's Relationship with Foreign Governments Central Banks, and International Organzations. (Kurzfassung des Kerry-Reports unter www.fas.org/irp/ congress/1992_rpt/bcci/).

O’Harrow, Robert Jr. (1998a): Data Firms getting too Personal?, in: Washington Post vom 8.März, A1, A18.

- (1998b): Laws on Use of Personal Data Form a Quilt With Many Holes?, in: Washington Post vom 9.März, A1, A12, A13.

Richelson Jeffrey T. und Desmond Ball (1990): The Ties That Bind: Intelligence Co-operation Between the UKUSA Countries, London.

Richelson, Jeffrey T. und Michael L. Evans (2001): National Security; Archive Electronic Briefing Book No 24, The National Security Agency Declassified. (Online-Version unter www.gwu.edu/ nsarchiv/NSAEBB23/index2.htm)

Sinclair, Scott (2000): GATS: How the WTO's New „Services“ Negotiations Threaten Democracy. Ottawa.

US General Accounting Office (1991): Money Laundering: The U.S. Government is Responding to the Problem. Washington DC, USGAO/NSIAD-91-130., p 13.

Whitaker, Reg (1992): Security and Intelligence in the Post-Cold-War Era in: New World Order? Socialist Register, herausgegeben von R. Miliband und L. Panitch, London.

Woodward, Veil (1987): The Secret Wars of the CIA 1981-1987, New York.

Wright, Steve (1998): Omega Foundation, STOA-Bericht des Europa-Parlaments vom 6. Januar bzw. in der aktualisierten Version vom September unter www.iptvreports.mcmail.com/ ic2kreport.htm. 